\title{
470 - Improving homeostatic systems and healthy longevity by intergenerational relationships: Evidences from a translational neuroscience approach
}

Díaz-Del Cerro $E^{1}$; Ceprián $\mathrm{N}^{1}$; Félix $\mathrm{J}^{1}$; Gimenez-Llort $\mathrm{L}^{3,4}$; De la Fuente $\mathrm{M}^{1,2, *}$

${ }^{1}$ Department of Genetics, Physiology and Microbiology (Unity of Animal Physiology), Faculty of Biology, Complutense University of Madrid (UCM), Madrid, Spain.

${ }^{2}$ Institute of Investigation 12 de Octubre (i+12), Madrid, Spain.

${ }^{3}$ Department of Psychiatry and Forensic Medicine, School of Medicine, Universitat Autònoma de Barcelona, Barcelona, Spain

${ }^{4}$ Institute of Neuroscience, School of Medicine, Universitat Autònoma de Barcelona, Barcelona, Spain

The aging process is associated with a deterioration of the physiological systems, especially the homeostatic (nervous, immune and endocrine) systems with the consequent increase in morbidity and mortality. With the aging population, increasing number of studies focus on lifestyle interventions to slow down these aging derangements. Here, animal models can be useful to assess their long-term effects and potential value taking into advantage their shorter life span. In a previous work, old animals beneficed of 2 months of continuous cohabitation with adult, with improvements on behaviour, immune function and redox state as well as a higher longevity. However, their adult counterparts showed impairments in these parameters. In the present study, this social strategy was modified with the aim to improve the homeostatic systems in both the old and the adult animals.) Animals of the experimental group with "two old ICR-CD1 female mice cohabiting 15 minutes each day for 2 months with five adult mic" were studied and compared to adult and old controls. After this time, mice were submitted to a behavioural battery of tests to analyse their sensorimotor abilities, anxiety-like behaviours, and exploratory capacities. Peritoneal leukocytes were collected and several immune functions, oxidative and inflammatory stress parameters as well as catecholamine concentrations were assessed. When the adult mice reached old age the same parameters were again analysed. The life span of each animal was also recorded. Several mice of each group were sacrificed to obtain plasma and the hormone oxytocin was evaluated. The results show that old mice presented an improvement of behavioural capacities, immune functions and lower oxidative and inflammatory stress after the two months of social interaction with adult animals, and consequently they exhibited an extended life span. Adult mice, in general, did not show any changes after social interaction, but when they achieved old age an improvement of all the parameters studied and of longevity was observed in comparison with those mice that never had a social interaction with old animals. In conclusion, a short social interaction between old and adult individuals can be an excellent strategy for improving in both the health state and longevity. 